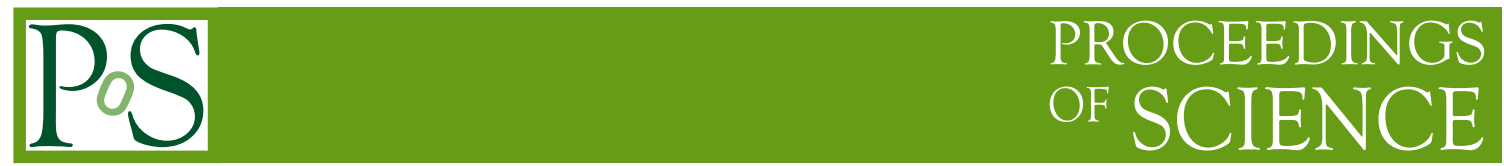

\title{
Performance of the LHCb Vertex Detector
}

\author{
Kazuyoshi Carvalho Akiba*† \\ Nikhef \\ Nikhef, Science Park 105, 1098 XG Amsterdam, The Netherlands \\ E-mail: kazuyoshi.akiba@cern.ch
}

This paper describes the design and commissioning of the LHCb Vertex detector (VELO). It shows preliminary results on efficiency (in general better than 95\%) and precision (from 8 to 30 $\mu \mathrm{m})$ using particles from one of the LHC injections tests. This paper also discusses tests with a high particle rate crossing a VELO sensor. These tests were performed in order to emulate an incident with the LHC beam.

9th International Conference on Large Scale Applications and Radiation Hardness of Semiconductor Detectors

30 September - 2 October 2009

Florence, Italy

\footnotetext{
*Speaker.

$\dagger$ on behalf of the LHCb VELO group
} 


\section{Introduction}

The LHCb experiment [1] is designed to measure $\mathrm{CP}$ violation and probe for physics beyond the Standard Model by studying the decays of b-hadrons produced in the $\mathrm{p}$ - $\mathrm{p}$ collisions provided by the Large Hadron Collider (LHC) at CERN, Geneva. The VErtex LOcator (VELO) is LHCb's primary tracking detector and it is used to reconstruct primary and secondary vertices, and measure the impact parameter with the high precision required to separate the long-lived B particles from the prompt hadronic background. The VELO is situated in an extreme radiation environment $8 \mathrm{~mm}$ from the beam during data taking and $30 \mathrm{~mm}$ from the beam during beam injection. The detector is operated in vacuum and has a dedicated bi-phase $\mathrm{CO}_{2}$ cooling system. In the following section the VELO system is shortly described, a more detailed description is found in [1].

\section{VELO}

The VELO is a silicon micro strip detector comprised of 2 retractable halves each containing 21 modules with $\mathrm{R}$ and $\phi$ measuring silicon sensors. The sensors are made of $\mathrm{n}$-strips implanted on a n-bulk with a double metal layer tracing the signals out. The front-end electronics operates at a $40 \mathrm{MHz}$ clock and is read out at a maximum rate of $1.1 \mathrm{MHz}$. The modules were designed to withstand high radiation doses $\left(1.3 \times 10^{14} \mathrm{n}_{\mathrm{eq}} \mathrm{cm}^{-2} / \mathrm{year}\right)$. A full replacement is foreseen to be installed after 3 years of nominal LHC operation.

The halves are enclosed in a secondary vacuum $\left(<10^{-4}\right.$ mbar) which is separated from the primary LHC vacuum $\left(<10^{-7}\right.$ mbar $)$ by a corrugated aluminum foil of $300 \mu \mathrm{m}$ thickness. The foil is specially designed to minimize the RF pickup onto the front-end and the inactive material before the first measured point. Active cooling is crucial for the operation in vacuum as there is no heat dissipation by convection. Furthermore, the final operational temperature in the silicon sensors was decided to be around $-10^{\circ} \mathrm{C}$, which requires a cooling set point of around $-30^{\circ} \mathrm{C}$. The cooling is provided by liquid $\mathrm{CO}_{2}$ which evaporates inside the cooling blocks attached to the hybrids. The cooling system of the VELO is described in [2]. The heat conduction inside the hybrid is improved by a layer of TPG (Thermal Pyrolytic Graphite) added to its construction. Further details to the module design can be found in [1].

The sensors, $\mathrm{R}$ and $\phi$, are composed by $300 \mu \mathrm{m}$ thick semi-circular silicon with 2048 strips and an overall radius of $4.2 \mathrm{~cm}$. Each sensor is glued to a double sided hybrid that supports the basic readout circuitry and provides power and mechanical support to the front-end chips (Beetles) which can each read out 128 strips. The hybrid requires 16 chips on each side to read out the sensors. The hybrid is then attached to a carbon fiber paddle to form a module as shown in Fig. 1

The modules are mounted onto a base plate and then connected to the cooling blocks. The complete bases are placed into the vacuum tank and inside the RF-shielding boxes. The whole system was completely assembled, tested and installed by October 2007.

The VELO sensors are readout via kapton cables connected to the hybrids and then into vacuum feed-through connectors which provide electrical connections to the outside vacuum region. The signals from the sensor are read out in analogue mode and repeated over $60 \mathrm{~m}$ in copper differential lines up to the radiation shielded barracks. The read out cables for each sensor are connected to a TELL1 (Trigger Electronics Level 1) board, where the signals are digitized, processed and 


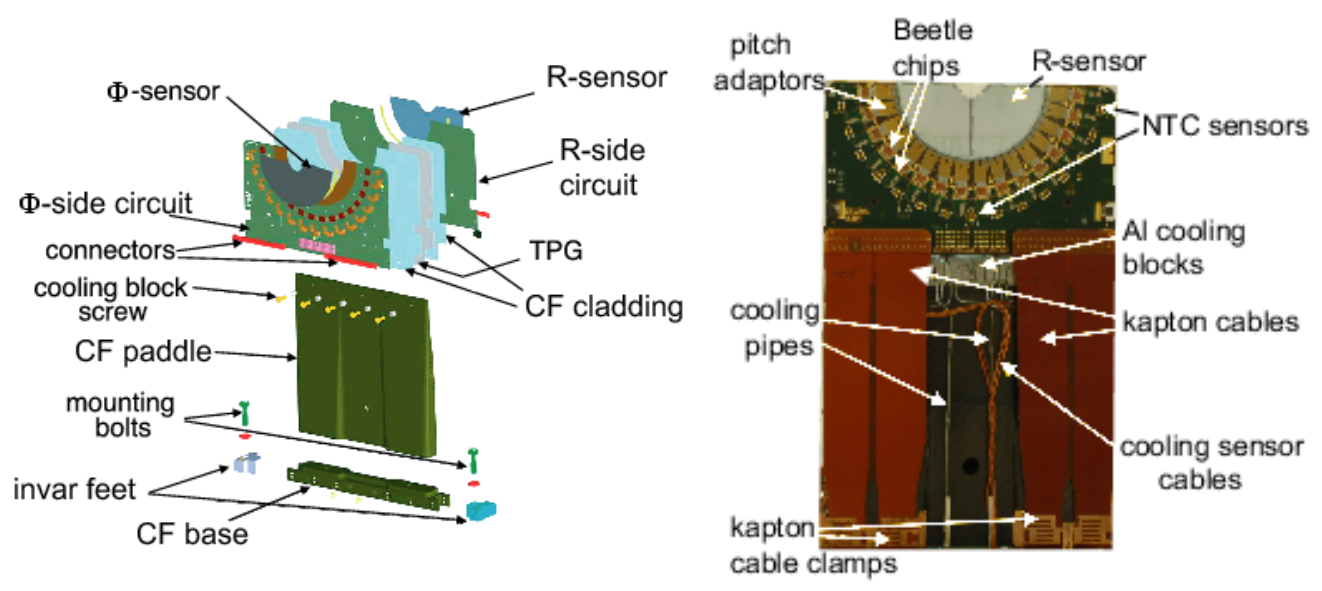

Figure 1: Principal components of a module (left) and a photograph of a mounted module (right).

zero-suppressed. The TELL1 is able to accept and read out the events at the maximum L0-Trigger frequency of 1.1 MHz. The TELL1 boards eventually send the VELO data through a Giga bit Ethernet connection to the LHCb's Event Filter Farm, where the High Level Trigger (HLT) takes place.

\section{Commissioning}

The commissioning of the VELO detector started right after its installation in 2007. Several power up and data acquisition tests were performed in 2008, including an individual module power up and readout as well as a full collective readout test. In every scenario the noise is measured and compared to the noise at previous stages of construction, burn-in, and assembly. Test pulses are used to assess overall signal to noise and synchronization of the front-end to the readout boards. Finally, full system tests were carried out in June 2008 showing an overall number of operational channels close to $99.2 \%$ of the total.

\section{Commissioning with Particles}

The final commissioning and timing of the detector cannot be performed using cosmic ray particles due to the small acceptance and the orientation of the silicon sensors. The LHC injection tests between August 2008 and June 2009 were used to commission the detector with particles. In the LHC synchronization tests protons were steered from the SPS via a transfer line to an absorber called the Transfer line External beam Dump (TED). located 340 m downstream of LHCb. Single bunches with varying intensities were shot into the absorber every $48 \mathrm{~s}$ and this led, on average, to 7 clusters per sensor and 10 tracks per event.

The first step was to find the optimal timing of the detector. A double Gaussian function to fit the front-end pulse shape as a function of time was used. Sampling points were obtained from the consecutive trigger samples (spaced by $25 \mathrm{~ns}$ ) and finer steps (of $6.5 \mathrm{~ns}$ ) by moving the clocks of the front-end Beetle chips together with those of the readout boards (TELL1). In this way the 

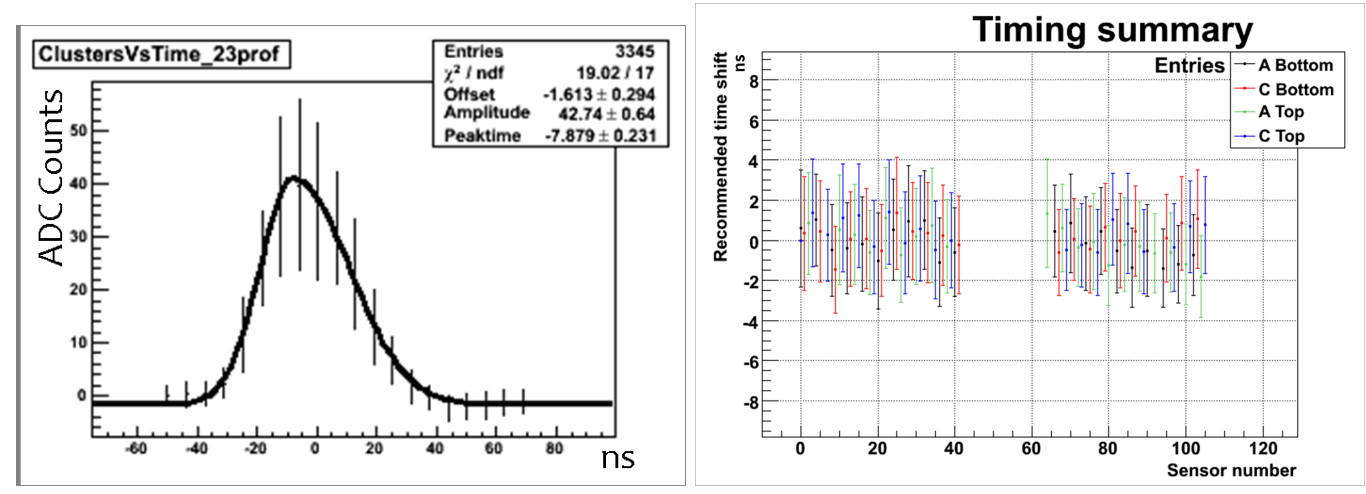

Figure 2: The plot on the left shows an example of the pulse shape obtained with the timing scan and the function fitted to the data points (solid line). The plot on the right shows the timing shift required to align the sensors after the setting from the scan was applied.

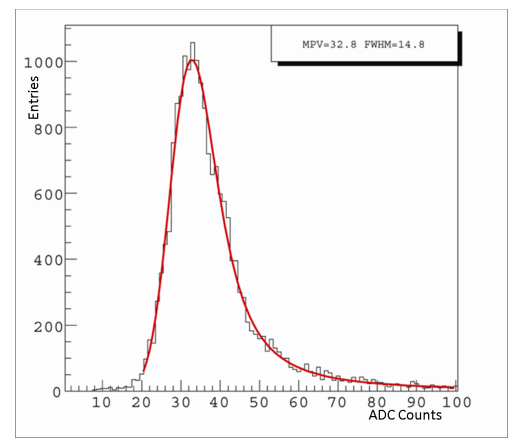

Figure 3: Cluster ADC sum data fitted with a Landau convoluted with a Gaussian for one of the R-measuring sensors.

timing of the detector can be tuned with a precision better than $1 \mathrm{~ns}$. Figure 2 shows the frontend pulse scanned over time and fitted to the double Gaussian function. The resulting setting was implemented and then checked by acquiring data with $25 \mathrm{~ns}$ spaced data points. The result of the checks is also shown in Figure 2 and the still remaining timing shifts for each sensor are compatible with zero.

Once the detector timing was tuned, other studies could be performed. Results on the signal, hit resolution, and efficiency are shown in figures 3, 4 and 5 respectively. The hit resolution for TED tracks, from 8 to $30 \mu \mathrm{m}$, is lower than that expected with LHC collision tracks because the TED tracks have their incidence close to orthogonal. The overall signal of the TED tracks show a very well behaved Landau convoluted with a Gaussian function and this leads to signal to noise values greater than 18 and 20 for $\mathrm{R}$ sensors and $\phi$ sensors respectively. The efficiency is estimated by using an interpolation method that requires at least the first and the last measured points of the track to be present. Since the efficiency is measured requiring hits on a few sensors in order to make the interpolation, it is called pseudo-efficiency. The pseudo-efficiency measured in this way is generally above $95 \%$ as seens in the Fig. 5 . 


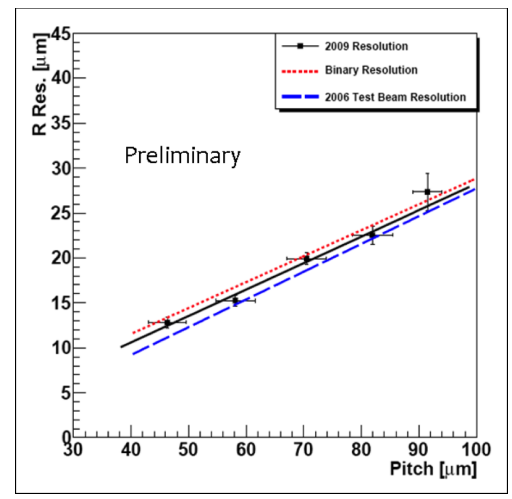

Figure 4: Preliminary results on the resolution of the VELO detectors with data from the TED run.

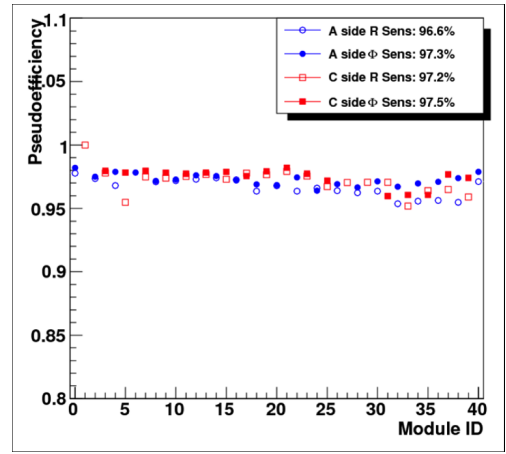

Figure 5: Efficiency of the VELO sensors measured with tracks from the TED run.

\section{High Rate Tests}

The LHC introduces an unprecedented stored beam energy which is 2 to 3 orders of magnitude larger than the previous colliders (Tevatron, LEP). From past experience and literature it is known that beam incidents can induce damage in silicon detectors, but little is known about the behavior of the silicon detectors under those circumstances. Other experiments (ATLAS, CMS) have already carried out tests with high particle fluxes [3], [4]. Since the VELO detector sits very close to the beam during the normal data taking even small beam excursions could hit the sensors. A study of possible damage from high particle fluxes was made by putting a VELO module into the PS booster beam and is described in this section.

\subsection{Setup}

A spare VELO module was mounted inside a light sealed box with connections for a vortex tube cooling system [5]. Each side of the module was individually powered by standard VELO service boards. The bias voltage for each sensor was provided by independent high voltage power supplies controlled by a computer in the control room. Each sensor had half of its chips configured and all of them were read out by a single TELL1 readout board. The configuration of the front-end 


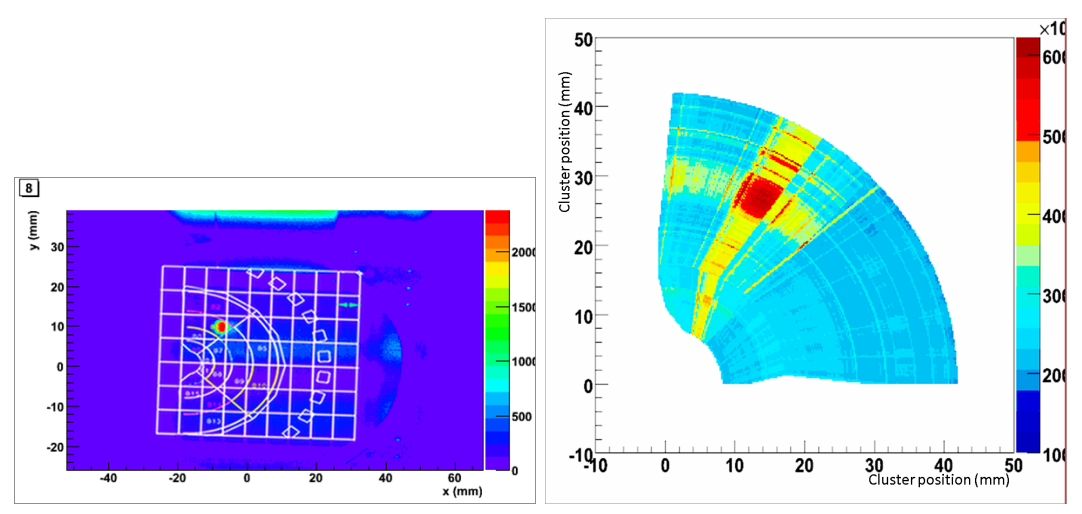

Figure 6: A picture of the viewer screen (left) taken while exposed to a shot and a plot of the combination of $\mathrm{R}$ and $\phi$ clusters (right). These are the two independent methods to measure the position of the incident beam. The two figures are put together for comparison, althought they do not show the same measured event.

chips was provided by the TELL1 board with some adapter card. A linux PC in the control room performed the acquisition of the events coming from the TELL1 board.

An aluminum oxide screen was mounted on top of the encapsulating box and was viewed with a radiation hard camera installed in the beam area. Figure 6 shows the light captured by the camera during one of the shots. These images provide an independent measurement of the position of the beam, and were used to point the beam when attempting to hit the front-end chips. In the Figure 6, right, the picture of the fluorescent screen is confronted to a combination of the $\mathrm{R}$ and $\phi$ clusters with hits during the same exposure to the beam. The plot shows a higher concentration of clusters around the beam spot also observed in the fluorescent screen. The combination of the clusters is shown in the units of mm. Finally, a retractable system was set up in order to move the sensor into the beam acceptance to absorb the shots and out to make noise and leakage current measurements away from the beam.

\subsection{Measurements}

Several beam shots were taken with different intensities and detector states. A shot is defined as one exposure to the particle beam. The bunch has a lengh varying from 20 to $60 \mathrm{~ns}$ (RMS), depending on the intensity, and a frequency of one per minute. Each shot contains only one bunch.

In between the shots all the functionality of both sides of the module was checked, and noise data were acquired. Shots were taken with all the combinations of the detector bias voltage on or off and front-end chips on or off.

\subsubsection{Shots on the Sensor}

A total of 31 shots with increasing intensities from $2.5 \times 10^{9}$ protons/bunch, to $2 \times 10^{10}, 2 \times$ $10^{11}$ and $2 \times 10^{12}$, up to a maximum of $\approx 9 \times 10^{12}$ protons/bunch was acquired following the systematic set of checks in each stage. The module survived this round with no measurable damage due to the shots. The leakage currents and the noise levels remained roughly the same. 


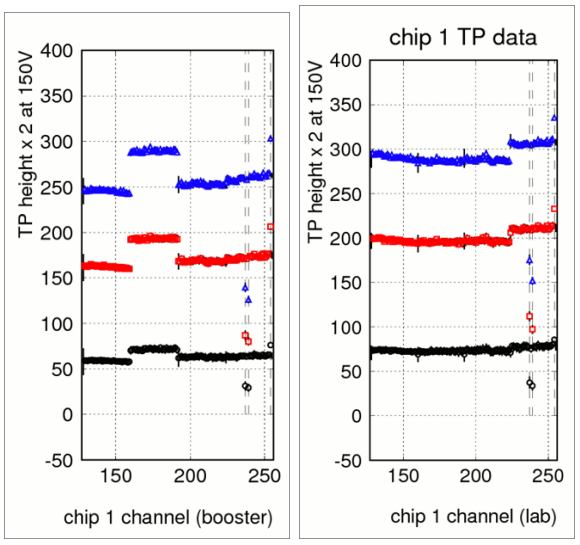

Figure 7: Test pulse amplitudes before (on the left) and after (on the right) the exposure to beam. The three discrete levels show the different charge injected in the pulses (1, 3 and 5 MIPs). The jumps in the levels of the pulses are due to the difference in the gain of the readout systems in the lab and at the booster site. The two channels, marked with a vertical dashed line, show shorted strips, consistent to the module characteristics measured when the module was built.

\subsubsection{Shots on the front-end chips}

The second set of measurements was directed at the front-end chips. Several shots with intensities from $2.5 \times 10^{9}$ protons/bunch, up to $2 \times 10^{11}$, protons/bunch were aimed at a few of the front-end chips. The intensities of these shots were increased gradually in the same way as for the ones on the sensors. One chip in particular was exposed to several shots with the intensity of $2 \times 10^{11}$ protons/bunch, with no obvious damage nor problem detected during the runs. By the end of the runs all the chips of the module could still be configured and the noise was compatible with the measurements before the exposure.

\subsection{Summary of Results}

No clear damage was found due to the high particle intensity shots. The noise levels, measured in situ before and after the shots, are within typical values and the leakage current does not show any increase above what is expected due to radiation. Test pulse scans were performed before and after the exposure to beam where each of the channels were individually pulsed. Figure 7 shows the overal results of these scans where the 3 levels of ADC counts stand for the injected charge equivalent to 1, 3 and 5 MIPs. The jumps on the levels are explained by the different gain in the different locations where the data were acquired. A few lower points are due to shorted strips, known from the construction. The total number of dead and shorted strips remained the same throughout the tests.

The module was sent back to Liverpool, where it was assembled, where it is subjected to a detailed laser scan. The first infrared pictures (see Fig. 8) show no hot spots on the sensors, which indicates that no pinholes were produced by the beam. 


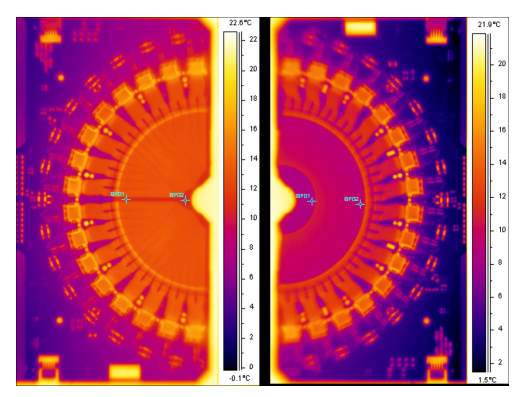

Figure 8: Thermal pictures taken of the module subjected to the high rate tests. In this picture the module is fully powered and biased. There is no sign of hot spots.

\section{Summary}

The LHCb VELO detector is successfully commissioned and fully tested. Beam induced tracks have been recorded during several opportunities and these particles were used to perform spatial and time alignment for the detector.

The tests with high particle fluxes were unable to show any clear beam induced damage on the VELO detectors nor on the front-end chips. Further analysis of the data taken during those tests is ongoing to try to identify any measurable effect caused by the beam shots.

\section{References}

[1] The LHCb Collaboration, The LHCb Detector at the LHC, JINST 3, S08005

[2] M. Van Beuzekom, B. Verlaat,A. Van Lysebetten. $\mathrm{CO}_{2}$ cooling experience (LHCb). The 16th International Workshop on Vertex detectors Lake Placid, NY, USA http://pos.sissa.it//archive/conferences/057/009/Vertex:202007_009.pdf

[3] A. Andreazza, K. Einsweiler, C. Gemme, L. Rossi, P. Sicho, Effect of accidental beam losses on the ATLAS pixel detector Nuclear Instruments and Methods in Physics Research A 565 (2006) 5054

[4] M. Fahrer, G. Dirkes, F. Hartmann, S. Heier, A. Macpherson, Th. Muller, Th. Weiler Beam-loss-induced electrical stress test on CMS Silicon Strip Modules Nuclear Instruments and Methods in Physics Research A 518 (2004) 328-330

[5] Some information on the vortex tube cooling system can be found on http://www.exair.com/en-US/Primary Navigation/Products/Vortex Tubes and Spot Cooling/Vortex Tubes/Pages/Vortex Tubes Home.aspx 